\title{
A study of an intensive home-based treatment program and its impact on admissions in an aged-care setting
}

\author{
Pam Callaly ${ }^{1,2}$, Jackie Kriz ${ }^{1}$, Jeanette Suters ${ }^{1}$, Kim Moon $^{1}$, Philip Dunn ${ }^{1}$, Margaret Henry ${ }^{2}$, Thomas Callaly ${ }^{1}$, Michael Berk ${ }^{1,2}$ \\ Community and Mental Health, Barwon Health, Geelong Victoria, Australia \\ ${ }^{2}$ Department of Clinical and Biomedical Sciences, University of Melbourne, Swanston Centre, Geelong, Victoria, Australia
}

\begin{abstract}
Objective: This study aims to evaluate an Intensive Home Based Program (IHBP) in terms of effects on admissions and length of stay. Method: The Brief Psychiatric Rating Scale (BPRS) and Mongomery Asberg Depression Rating Scale (MADRS) were completed on two patient cohorts, IHBP ( $n=65)$ and in-patients (IPS) ( $n=29)$. Hospital admissions and length of stay were also measured. Diagnostic and treatment data for patients were collected. Results: The clinical outcomes of a sample of patients who were treated on the IHBP were compared to those treated as IPS, and the impact on hospital admissions was considered. Clinical improvement was greater in the more severely ill IPS, but those treated on the IHBP also showed significant improvement. Initially, rates of hospital admissions decreased but have since increased as have admissions to the IHBP. Conclusion: The program is in its third year, is well received and utilised, is considered useful in providing early intervention and may have a role in preventing hospital admissions.
\end{abstract}

Key words: Intensive home based program, health services, admission, outpatient, inpatient, aged care.

Received: 09.06.2004

Accepted: 25.08.2004

For many years, community treatment options have been sought for people suffering acute psychiatric symptoms as an alternative to psychiatric hospital admission, which is costly and takes the patient away from community life. ${ }^{1}$ One type of community care that relies largely on making contact with the patient in their home rather than in mental health services is known by a variety of names including assertive or intensive case management ${ }^{2}$, and a growing body of research supports its efficacy. ${ }^{3}$

In the aged psychiatry setting, evidence supports the multidisciplinary, community based mental health team. ${ }^{4}$ When individually tailored packages of care have been implemented in the community for those with dementia, behavioural disturbance has decreased and there has been an improvement in carer well-being. ${ }^{5}$ Another study showed a reduction in the needs of older people with dementia and their carers when an intensive aged care service was integrated with social services. ${ }^{6}$

Encouraging results were also found when depressed elderly patients were treated by a community orientated aged care mental health service which aimed to reduce hospital length of stay and provide responsive follow-up. This study highlighted the importance of cooperation with other services such as primary health, other hospital specialists and social services. ${ }^{7}$ A study that compared normal gen-

\author{
Correspondence: \\ Professor M Berk,Department of Clinical and Biomedical Sciences, \\ University of Melbourne, Swanston Centre, PO Box 281, Geelong \\ Victoria 3220, Australia \\ email:mikebe@barwonhealth.org.au
}

eral practitioner care to individually tailored community nursing interventions in the primary care setting also found the latter to be more effective for elderly people with depression. ${ }^{8}$ In an elderly disabled population, community psychiatric treatment was found to be more effective than GP care alone in the treatment of depression. ${ }^{9}$

\section{The Barwon Health Aged Psychiatry Service}

The Aged Psychiatry Service (APS) is a community based multidisciplinary psychiatric service that serves approximately 37,000 people over the age of 65 within the Geelong and Colac regions of Victoria (Australia), a catchment area of a quarter of a million people. The target population are those with significant psychiatric disorder or with dementia with severe behavioural or psychiatric symptoms. A typical community service response includes assessment followed by advice, education, treatment, brief supportive therapy or case management, and this usually occurs in collaboration with the treating GP. All patients are case managed who require a full assessment by APS.

The APS was established in 1996 with one half time psychiatrist and two community psychiatric nurses, and over the past nine years has grown to a team with two half time psychiatrists, one psychiatric registrar and ten community case managers together with administration staff and a team coordinator. The APS has only had access to four acute psychiatric inpatient beds situated within an acute general adult psychiatric unit, but staff have long recognized that for nursing the frail elderly, this environment is not ideal.

Because of the low number of beds and the inappropriateness of the environment for the elderly it has been necessary, even before the introduction of the intensive house based programme (IHBP), 
for the APS to explore and support alternatives to admission to this unit, such as admission to private psychiatric or general hospitals with ongoing management by APS. When appropriate, alternatives such as short admissions to Supported Residential Services (SRS's), hostels or to a relative's home with intensive team support have been used. Additional support services accessed from both public and private agencies were sometimes also used and funded by APS or more commonly by the patient or their family.

\section{The Intensive Home-Based Program}

The Intensive Home Based Program (IHBP) was established in June 2000 to strengthen the existing community care practices. However, those at risk from severe psychosis, with suicidal intent, with an unclear diagnosis or comorbid medical problems, or those whose carer was unable to cope were still admitted to hospital. It was anticipated that those requiring the IHBP would include patients with poor treatment compliance, inadequate social supports, carer breakdown or an exacerbation of a chronic mental illness.

The IHBP provides intensive daily follow-up within a case management model following multidisciplinary assessment and is supported through a team approach. The case manager is accountable for care planning, monitoring and review of clients and for arranging and monitoring support services. A brokerage fund is available for the purchase of support services ranging from agency care workers who assist the patient in their home to time -limited supported residential accommodation options if the home environment is unsuitable. The GP retains their existing role with the patient, and the APS psychiatrist and case manager liase closely with them.

This study aims to evaluate the IHBP in terms of effects on admissions and length of stay. The clinical outcomes, using the Brief Psychiatric Rating Scale (BPRS) and Mongomery Asberg Depression Rating Scale (MADRS), of a sample of people who were on the IHBP were compared to in patients (IPs). The hypothesis of the intervention was that the IHBP would reduce inpatient admissions and length of stay while having a comparable clinical outcome.

\section{Method}

Rating scales were completed on 65 IHBP and 29 IP admissions between June 2000 and Feb 2002. The number and duration of psychiatric IP admissions of patients over 65 from 1998 to 2002 was measured. Case managers from the APS who were trained psychiatric nurses, occupational therapists or social workers conducted the study in a community setting.

\section{Participants}

Selection to either the IHBP or IP care was not randomised. Patients were typically selected for IP admission if they presented with risks associated with severe psychosis or suicide, unclear diagnosis, comorbid medical problems or carer inability to cope. Patients selected for the IHBP were more likely to have issues regarding treatment compliance, inadequate social supports, carer breakdown or chronic psychiatric illness. There was however substantial overlap between the clinical characteristics of the two groups.

Those on the IHBP received a wide range of services that enabled them to remain in the community during their psychiatric episode. Both public and private service providers were used including personal carers, housekeepers, and the temporary use of private supported accommodation facilities. These services were coordinated and monitored daily by the case manager, who liased with the patient, family, APS, GP and other involved agencies. This project was approved by Barwon Health and the Research and Ethics Committee.

\section{Measurements}

Case managers completed client profile forms on admission and discharge from both programs. Scales completed in all cases were a modified BPRS and the MADRS. Most of the assessments were completed by the same clinicians on both admission and discharge. These outcome measures were used on all patients in the study irrespective of diagnosis in order to have a standardised battery of measures applicable across the most common problems seen in the unit.

Retrospective data pertaining to the number of in-patient admissions, bed occupancy days and average length of stay of the elderly population from 1997 to 2002 was collected. The diagnoses and treatment for all of the patients who were case managed by the service was collected, and these were compared to the IPs and those who went on the IHBP.

\section{Results}

The sample (which was used to compare treatment outcomes) consisted of 65 subjects in the IHBP and 29 in the IP group. The median age in both groups was 73yr (range 64-93yr) ( $\mathrm{p}=0.602$; Mann Whitney test). There was no significant difference in gender across the two groups (IHBP: 26\% male, 74\% female; IP 17\% male, 83\% female; $\mathrm{p}=0.346$; Chi-square statistic). With regard to diagnosis (Figure 1), there were no differences between the groups $(\mathrm{p}=0.221)$.

On the BPRS scale, the mean difference (baseline minus endpoint scores) in the IHBP group was 7.65 (SD 9.74) and 17.5 (SD 10.9) in the IP group. This difference was highly significant $(\mathrm{p}=0.0000,2$ sample $\mathrm{t}$ test) (Figure 2). Similarly, On the MADRS scale (Figure 3), the mean difference (baseline minus endpoint scores)
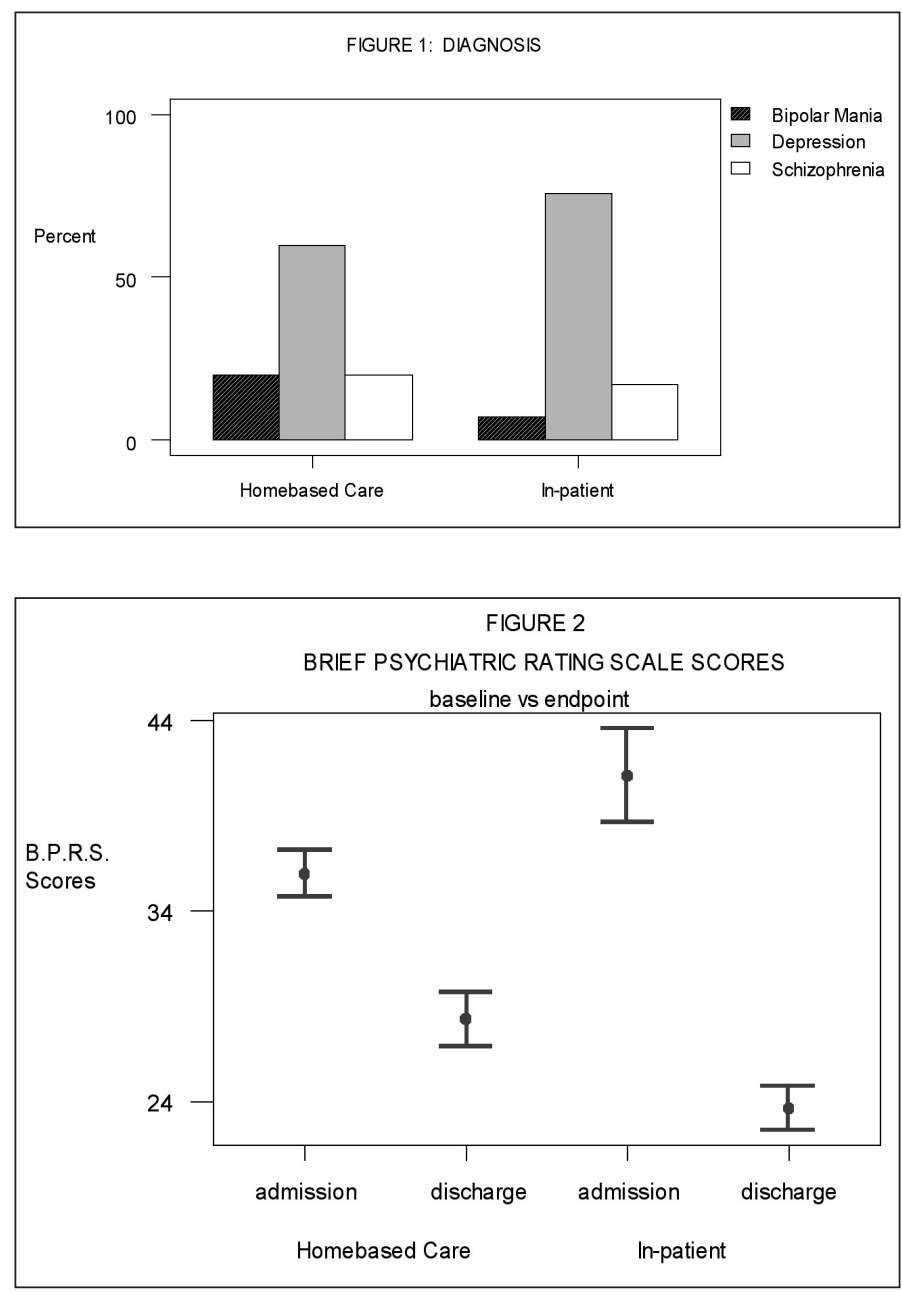


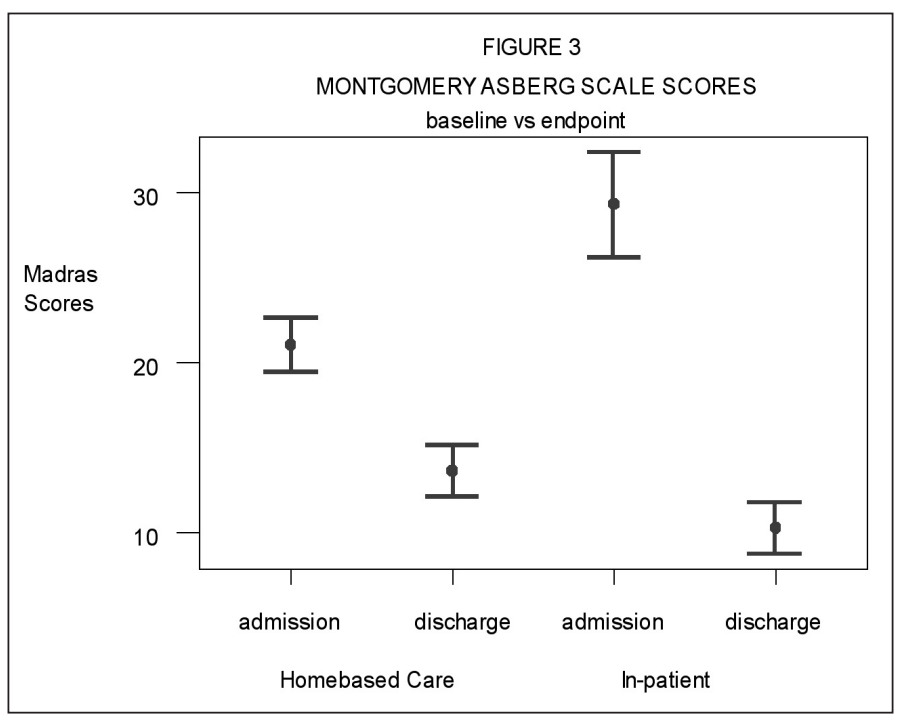

in the IHBP group was 7.40 (SD 11.5) and 19.0 (SD 14.8) in the IP group. This difference was again highly significant $(\mathrm{p}=0.0001,2$ sample $\mathrm{t}$ test)

\section{Effect on hospital admissions and length of stay}

With regard to the average length of stay in either IHBP or inpatient care, the median lengths were 13 days (Inter quartile range
5-21) and 16 days (Inter quartile range 7-31) respectively ( $\mathrm{p}=$ 0.164; Mann Whitney test).

In 1999, there were 53 IP admissions, using a total of 846 bed days, with the average length of stay being 15.96 days. In 2000, IP admissions were 65, using 913 bed days and the average length of stay was 14.04 days. The IHBP began in June of this year, but an extensive restructuring of services also occurred at this time, resulting in an increase of patients to the APS. In 2001, the first full year of the IHBP, admissions decreased to 40, using 855 bed days, with the average length of stay increasing to 21.37 days. 45 people were admitted to the IHBP, for an average of 11.95 days. By 2002, hospital admissions had increased to 67, using 1372 bed days and the average length of stay was 20.48 days. 64 patients were admitted to the IHBP for an average of 11.25 days (Table 1)

While $36 \%$ of all patients managed by APS suffered from depression, $39 \%$ of IPs and $60 \%$ of those on the IHBP suffered from depression. $42 \%$ of all case managed patients suffered with behavioural disturbance in dementia, but only approximately $20 \%$ of IPs and those on the IHBP had dementia. Anxiety disorders accounted for $16 \%$ of all case managed patients, with $24 \%$ of IPs and $30 \%$ of those on the IHBP suffering from anxiety disorders (Table 2). $5 \%$ of all case managed patients were treated with ECT, while $20 \%$ of IPs and $7 \%$ of those on the IHBP had ECT (Table 3).

\begin{tabular}{|l|l|l|l|l|l|l|}
\hline \multicolumn{2}{|c|}{ Table 1. Admissions of people over $\mathbf{6 5}$ to the psychiatric in-patient unit and the intensive home based program from $\mathbf{1 9 9 8}$ to 2002} \\
\hline Year & In-patient admissions & $\begin{array}{l}\text { Intensive home based } \\
\text { entrants }\end{array}$ & $\begin{array}{l}\text { Total in-patient } \\
\text { bed days }\end{array}$ & $\begin{array}{l}\text { Total intensive home } \\
\text { based days }\end{array}$ & $\begin{array}{l}\text { Average length of stay } \\
\text { for in-patient } \\
\text { admissions (days) }\end{array}$ & $\begin{array}{l}\text { Average length of time } \\
\text { on intensive home } \\
\text { based program (days) }\end{array}$ \\
\hline 1998 & 56 & & 738 & & 13.17 & \\
1999 & 53 & 846 & 276 & 15.96 \\
2000 & 65 & 913 & 538 & 21.37 & 13.8 \\
2001 & 40 & 855 & 720 & 20.48 & 11.95 \\
2002 & 67 & 64 & 1372 & & 11.25 \\
\hline
\end{tabular}

\begin{tabular}{|c|c|c|c|c|c|c|c|c|}
\hline Diagnosis & Schizophrenia & Depression & Dementia & $\begin{array}{l}\text { Bipolar and } \\
\text { schizoaffective } \\
\text { disorder }\end{array}$ & $\begin{array}{l}\text { Delusional } \\
\text { disorder and } \\
\text { psychosis }\end{array}$ & Anxiety & $\begin{array}{l}\text { Personality } \\
\text { Disorder }\end{array}$ & Delirium \\
\hline $\begin{array}{l}\text { All case managed } \\
\text { patients }\end{array}$ & 12 & $\begin{array}{l}\text { Mild 6 } \\
\text { Mod 22 } \\
\text { Severe } 8 \\
\text { Total } 36\end{array}$ & 42 & 5 & 8 & 16 & 5 & 5 \\
\hline In-patients & 18 & $\begin{array}{l}\text { Mild } 4 \\
\text { Mod } 11 \\
\text { Severe } 24 \\
\text { Total } 39\end{array}$ & 23 & 13 & 16 & 24 & 6 & None \\
\hline $\begin{array}{l}\text { Intensive Home } \\
\text { Based Program }\end{array}$ & 7 & $\begin{array}{l}\text { Mild } 4 \\
\text { Mod } 17 \\
\text { Severe } 39 \\
\text { Total } 60\end{array}$ & 21 & 13 & 20 & 30 & 4 & 4 \\
\hline
\end{tabular}

Table 3. Treatments given to patients case managed by APS during the study period 14/6/00 - 28/2/02 (\%)

\begin{tabular}{|l|l|l|l|l|}
\hline Medication & Antipsychotics & Antidepressants & Mood Stabilisers \\
\hline All case managed patients & 50 & 40 & 6 & 15 \\
In-patients & 73 & 36 & 45 & 13 \\
Intensive Home Based Program & 66 & 45 & 7 \\
\hline
\end{tabular}




\section{Limitations of the study}

The study was non-randomised across samples, selection to either program was based on severity criteria, and the sample size was small. The comparison of two very different groups is a limitation. Incomplete data entry is often an issue in audits. With the most severely ill patients potentially admitted to IP care, those on the IHBP were more likely to have chronic or less severe psychiatric illnesses. Non-blind assessment by raters with the possibility of bias is another limitation. $\mathrm{Pa}$ tients with a diagnosis of dementia were not included in the study, although they comprised approximately one fifth of the IPs and one fifth of the IHBP. The routine outcome measures used were also of limited utility in dementia, reducing the validity of including that cohort.

During the study period, restructuring of the service occurred, closer links with other agencies were established, staffing was increased and the service expanded to 7 days a week.

\section{Discussion}

IPs showed significantly greater clinical improvement than patients did on the IHBP, perhaps because more severely ill people were selected for IP treatment. Patients on both programs nevertheless showed significant clinical improvement. When there are higher scores on symptom rating scales there is much more room to move so one expects greater symptom reduction in more acutely ill patients.

The IHBP began in June 2000, and IP admissions rose dramatically in that year, although one of the aims of the program was to decrease IP admissions. This increase in hospital admissions was thought to be due to restructuring of the service when the Barwon Health network was formed, and many more patients came under the auspices of the APS. Marketing of the service was stepped up and staffing was increased.

However in 2001, IP admissions decreased markedly, but their length of stay increased, which may be a reflection of the less severely ill patients being treated on the IHBP. 45 patients were treated on the IHBP in that year.

2002 saw an increase to 67 IP admissions, while 64 patients were treated on the IHBP. Since 2000, IP admissions and IHBP admissions have increased. Staffing of the APS has remained at an increased level, and rapport with the GPs and other community agencies has continued to receive high priority within APS. This study suggests that the IHBP overall did not decrease number of admissions or length of stay. This may however be a result of other structural changes in the system. Of interest is the fact is the overall admissions to the ward increased in parallel with the IHBP treatment rate, suggesting that a general increase in service activity may be a factor.

With regard to diagnoses, the IHBP has been particularly useful in caring for those with depression and anxiety outside of the hospital setting. While $5 \%$ of all case managed patients and $7 \%$ of those on the IHBP had ECT, $20 \%$ of IPs had ECT which is expected as these are the most severely ill group.

A smaller percentage of those with behavioural disturbance in dementia have been treated on the IHBP or as IPs than have been case managed by the APS. There are two possible reasons for this. Firstly, a psychogeriatric unit (PGU) operates within the APS providing strategies and education to those caring for patients with severe behavioural disturbances associated with dementia. A separate budget allows for services to be provided to dementia sufferers and their carers. Secondly, dementia sufferers are often in hostel and nursing home accommodations and the staff of these facilities are educated to manage the behavioural disturbance.

The IHBP continues today, and while the service aims to eventually build a psychogeriatric in-patient assessment facility when funding becomes available, the IHBP will continue to operate and expand. The IHBP was originally intended as an alternative to IP care. However, it has become evident over time that is also used to prevent deterioration in people who may require IP care in the future. The extra level of care provided by the IHBP provides both an alternative to hospitalisation, as well as having an early intervention function.

\section{Conclusion}

The advent of the Intensive Home Based Program (IHBP) has allowed the APS to continue and build on an already existing model of home care where staff had previously attempted to provide intensive home care where necessary. Although this was largely successful, it was often complex, limited and constrained by the lack of a formal structure with funding provided specifically for intensive home care. The IHBP has considerably reduced the emotional and financial burden that this unfunded model was placing on patients and their families.

\section{References}

1. Wykes $T$, Leese M, Taylor R, Phelan M. Effects of community services on disability symptoms. PriSM Psychosis Study 4. Brit J Psychiatry 1998; 173:385-390.

2. Hambridge JA, Rosen A. Assertive community treatment for the seriously mentally ill in suburban Sydney: a programme description and evaluation. Austral New Zeal J Psychiatry 1994; 28:438-445.

3. Rosen A, Teeson M. Does case management work? The evidence and the abuse of evidence-based medicine. Austral New Zeal J Psychiatry 2001; 35:731-746.

4. Bartels S, Aricca RD, Oxman T, Schneider LS, Arean P A, Alexopoulos GS, Jeste DV. Evidence-based practices in geriatric mental health care. Psychiatric Serv 2002; 53:1419-1431.

5. Hinchcliffe AC, Hyman IL, Blizard B, Livingston G. Behavioural complications of dementia - can they be treated? Int J Geriatr Psychiatry 1995; 10:839-847.

6. Challis D, von Abendorff R, Brown P, Chesterman J, Hughes J. Care Management, dementia care and specialist mental health services: an evaluation. Int J Geriatr Psychiatry 2002; 17:315-325.

7. Baldwin RC, Jolley. The prognosis of depression in old age. Brit $J$ Psychiatry 1986; 149:574-583.

8. Blanchard MR, Waterreus A, Mann AH. The effect of primary care nurse intervention upon older people screened as depressed. Int J Geriatr Psychiatry 1995; 10:289-298.

9. Banerjee S, Shamash K, Macdonald AJD, Mann AH. Randomised controlled trial of effect of intervention by psychogeriatric team on depression in frail elderly people at home. Brit Med J 1996; 313:7064; Health Module pg. 1058. 


\title{
Commentary
}

\author{
Lennart Eriksson
}

Consultant psychiatrist to Alzheimer's South Africa

This study, by Pam Callaly and her team, highlights the need for both community services and an in-patient facility designated to care of persons over the age of 65 years. The addition of an additional specialised service tier, in the form of an intensive home based program (IHBP), illustrates the complexity of providing a service to the severely mentally ill elderly population. The study sets out to explore the benefit of adding a specialised service in the form of an Intensive Home Based Program to an already existing service - Aged Psychiatry Service (APS).

The cry of politicians and economists alike is to avoid inpatient care "at all cost". Therefore, an important component of the study, as confirmed by the study hypothesis, was to lend support to the idea that an "Intensive home based programme would reduce in-patient admissions and length of stay while having a comparable clinical outcome". With the successful testing of this hypothesis both sides of the clinical divide would be happily vindicated!

The study fails to demonstrate that the introduction of an IHBP will reduce the number of elderly severely mentally ill persons requiring in-patient treatment. In 199953 severely mentally ill persons were admitted for in-patient treatment. In 2002, a year after the introduction of the IHBP, 67 admissions took place. Bed days increased from 846 in 1999 to 1372 bed days in 2002. This news is hardly going to please any policy maker. To further point to the reality of the situation "on the ground" was the need to expand the IHBP staffing and make the service available seven days a week. No music to the ears of any economist! To add to this is the IHBP's intention to expand their services to include an in-patient psycho-geriatric assessment facility. From a clinical perspective this is an essential and logical vision if the intention is to provide an adequate service to the severely ill over the age of 65 years. An expanded service will likely soon be fully utilised in a country with a burgeoning elderly population.

\footnotetext{
Correspondence:

Dr L Eriksson, P0 Box 14, Pennington, 4184, Kwazulu Natal, South Africa email: lennarte@iafrica.com
}

So how is this study relevant to the South African situation? The Australian understanding of, and policy support for, an integrated service designated and designed for the care of the severely mentally ill over the age of 65 years is to be applauded. A psychiatric service that acknowledges a multi-disciplinary mental health team intimately linked to social services, primary health and hospital specialists - whilst still including the general practitioner in the loop - is a model that we would do well to pursue and implement. The commitment to adequate funding and staffing of a community service is only given lip service in South Africa. This study demonstrates that even when a designated service to the elderly is operational in the community (Aged Psychiatry Service (APS)) the addition of a further service (IHBP) only served to highlight the need for more staff and more specialised services. Such is the need in order to supply a quality care for the elderly.

The mechanics of the study has, by admission, many shortcomings. These shortcomings however do not detract from the final conclusions. The study, without referring back to the initial hypothesis, concludes with the statement that "the IHBP has considerably reduced the emotional and financial burden that the unfunded Adult Psychiatric Service (APS) was placing on patients and their families". The extent to which the APS is "unfunded" is not discussed. Nevertheless, this concluding remark, clearly designed to alert policy makers and economists to the need for adequate funding and staffing of both community based services and an in-patient facility, allows the study to achieve an important goal.

In South Africa we have no political will to allocate financial or human resources to the establishment of even a skeletal community based case management service for the severely mentally ill elderly members of our community. As our young population greys this study must alert us, as mental health professionals, and, hopefully, also policy makers to the urgent need for a multidisciplinary community service supported by specialist medical staff who are attached to designated in-patient facility. Neuropsychiatric problems, with which the elderly most often present, cannot be adequately assessed or managed by either a community service alone or a community service who's only referral source is a primary health facility. 